\title{
Reappraisal modulates the electrocortical response to unpleasant pictures
}

\author{
GREG HAJCAK \\ Vrije Universiteit, Amsterdam, The Netherlands \\ and Stony Brook University, Stony Brook, New York \\ and \\ SANDER NIEUWENHUIS \\ Vrije Universiteit, Amsterdam, The Netherlands
}

\begin{abstract}
Cognitive strategies such as reappraisal reduce the intensity of negative experience and brain activity that is sensitive to emotional salience. The time course of reappraisal-related neural modulation remains unclear, and it is unknown whether the electrocortical response to emotional stimuli is sensitive to reappraisal. Event-related brain potentials were recorded first while participants passively viewed pleasant, unpleasant, and neutral pictures, and then during an emotion regulation block in which participants were instructed to attend to or reappraise unpleasant pictures. The late positive potential (LPP) was enhanced for pleasant and unpleasant pictures in the passive viewing block, and reappraisal resulted in a reliably reduced LPP - a protracted modulation that began $200 \mathrm{msec}$ after stimulus onset. The degree of LPP modulation was positively related to reductions in the self-reported emotional intensity that followed emotion regulation instructions. These results indicate that reappraisal modulates early electrocortical activity that is related to emotional salience, and that the LPP is a useful tool for studying emotion regulation.
\end{abstract}

The influence of cognitive control over emotional responses has been labeled emotion regulation and has been defined as "the extrinsic and intrinsic processes responsible for monitoring, evaluating, and modifying emotional reactions, especially their intensity and temporal features" (Thompson, 1994). Understanding emotion regulation is especially important insofar as the inability to successfully regulate emotional responses plays an important role in affective disorders as well as most personality disorders (American Psychiatric Association, 1994).

Gross (1998) distinguishes between response- and antecedent-focused emotion regulation strategies. The former refers to processes such as expressive suppression, which involves modifying an emotional response once it is already activated, whereas the latter describes strategies to modify emotional responses before they become activated. An example of antecedent-focused emotion regulation is the cognitive reappraisal strategy, which involves interpreting a situation so that its emotional impact is altered (Gross \& John, 2003). Reappraisal is rooted in the work of Lazarus, who demonstrated that the interpre-

This research was supported in part by NIMH predoctoral fellowship Grant MH069047 to G.H. and by a grant of the Netherlands Organization for Scientific Research to S.N. We thank Niels Alting von Geusau for assistance with the data collection and analysis. S.N. is now at the Department of Psychology, Leiden University. Correspondence concerning this article should be sent to G. Hajcak, Department of Psychology, Stony Brook University, Stony Brook, NY 11794-2500 (e-mail: greg .hajcak@stonybrook.edu). tation of a stimulus influences the subsequent emotional response (Lazarus, 1991). In comparison with expressive suppression, reappraisal appears to be a more effective method of emotion regulation; only reappraisal has been shown to decrease self-reported negative emotional experience (Gross, 2002). The notion that reappraisal reduces negative emotional experience is similar to the idea that is the basis for cognitive therapy - that cognition can significantly influence emotion.

Recent studies have begun to shed light on the neural correlates of emotion regulation. Using functional magnetic resonance imaging (fMRI), Ochsner and colleagues found that reappraisal of unpleasant stimuli was associated with increased activation of the prefrontal cortex and decreased activation of the amygdala (Ochsner, Bunge, Gross, \& Gabrieli, 2002; Ochsner et al., 2004; see also Phan et al., 2005). Similar results have been observed in studies on the effects of voluntary suppression of responses to emotional stimuli (Beauregard, Lévesque, \& Bourgouin, 2001; Lévesque et al., 2003). Overall, the results are consistent with the idea that certain areas of the prefrontal cortex subserve cognitive control processes that down-regulate activity in the amygdala and other neural structures that are involved in coding emotionally salient stimuli (Anderson \& Phelps, 2001; Whalen et al., 1998).

As pointed out by Thompson (1994), an important aspect of emotion regulation concerns the time course of changes induced by emotional responses. With regard to emotionrelated brain activity, it is possible that reappraisal takes effect early and dampens the entire emotion-related response 
to unpleasant stimuli. However, another possibility is that unpleasant stimuli first elicit an automatic emotional response and then a reappraisal-related modulation of this initial response. Although fMRI is important for investigating the neural structures implicated in emotion regulation, its poor temporal resolution renders it unsuitable for studying the time course of reappraisal-induced neural modulations.

A more useful method for studying the temporal characteristics of reappraisal-related processes might be provided by electroencephalography. Specifically, electroencephalography studies have revealed that the late positive potential (LPP), a component of the event-related potential (ERP), differs substantially between emotionally salient and neutral stimuli (Cuthbert, Schupp, Bradley, Birbaumer, \& Lang, 2000; Keil et al., 2002; Lang, Bradley, \& Cuthbert, 1997; Schupp et al., 2000; Schupp, Junghöfer, Weike, \& Hamm, 2003). The LPP is a positive slow modulation of the ERP with a posterior midline scalp distribution and an onset around $250 \mathrm{msec}$ after stimulus presentation. Importantly, the LPP is highly sensitive to the emotional intensity of stimuli and is larger for both pleasant and unpleasant than for neutral stimuli (Cuthbert et al., 2000; Keil et al., 2002; Lang et al., 1997; Schupp et al., 2000; Schupp et al., 2003). Thus, given the functional sensitivity of the LPP and the excellent temporal resolution of ERPs, the LPP may be ideal for studying the time course of emotion regulation. Indeed, preliminary data suggest that the LPP is reduced under conditions of voluntary suppression of negative emotion (Moser, Hajcak, Bukay, \& Simons, 2006). In the present study, we set out to determine whether reappraisal would impact the magnitude of the LPP, and to examine the temporal characteristics of this modulation.

To this end, we measured the LPP in an experiment in which the participants were instructed to reappraise unpleasant stimuli. In the first stage of the experiment, participants passively viewed pleasant, neutral, and unpleasant pictures, a process we used to establish the effect of emotional intensity on the LPP. In the second stage of the experiment, we examined the effects of reappraisal on the LPP using an experimental paradigm similar to that employed in previous fMRI studies (Ochsner et al., 2002; Phan et al., 2005). Specifically, participants once again viewed each unpleasant picture, with instructions either to reinterpret the meaning of the picture (experimental condition) or to simply attend to it (control condition). A comparison of the LPPs associated with these conditions allowed us to examine the time course of the neural modulation related to the reappraisal of unpleasant stimuli. Finally, following each trial, participants rated the intensity of their emotional response; this process allowed us to relate reappraisal-induced changes in the LPP to self-reported differences in emotional intensity.

\section{METHOD}

\section{Participants}

Fourteen participants (11 female) were recruited from the Vrije Universiteit community via e-mail and advertisements. All participants were paid $€ 12$ (approximately $\$ 14$ U.S.) for their participation. Written informed consent was obtained from all the participants, and the experiment was approved by the research ethics committee of the Vrije Universiteit medical center.

\section{Stimuli}

A total of 120 pictures was selected from the International Affective Picture System (IAPS; Lang, Bradley, \& Cuthbert, 1999). Of these, 40 depicted unpleasant events (e.g., threatening scenes), 40 pleasant events (e.g., smiling families, nudes), and 40 neutral events (e.g., household objects, leaves, trees). ${ }^{1}$ The three categories differed in their normative ratings of valence (means of 7.01, 5.03, and 2.52, for pleasant, neutral, and unpleasant picture content, respectively). Additionally, the emotional pictures scored reliably higher on normative arousal ratings (means of 5.49, 2.74, and 6.03, for pleasant, neutral, and unpleasant picture content, respectively). These means are similar to those reported in previous studies of the LPP (Cuthbert et al., 2000; Keil et al., 2002; Schupp et al., 2003).

The task was administered on a Pentium I computer, using Presentation software (Neurobehavioral Systems, Inc.) to control the presentation and timing of all stimuli. Each picture was displayed in color and occupied the entirety of the 18 -in. monitor. At a viewing distance of approximately $65 \mathrm{~cm}$, each picture occupied nearly $25^{\circ}$ of visual angle, vertically, and $30^{\circ}$, horizontally.

\section{Procedure}

The experiment consisted of two blocks of trials: a passive viewing block followed by an emotion regulation block. The purpose of the passive viewing block was to establish the basic effect of emotional salience on the LPP. To this end, the participants were instructed to simply view the 40 pleasant, 40 unpleasant, and 40 neutral IAPS pictures. The 120 pictures were presented in random order for 1,000 msec each, with a 1,500-msec blank screen between picture presentations.

In the emotion regulation block, the participants were given reappraisal instructions that were intended to closely parallel the study by Ochsner et al. (2002). The experimenter explained the concept of reappraisal as reinterpreting an unpleasant picture so that it no longer elicited a negative response. The participants were first given several examples of this process, which was illustrated with unpleasant pictures that were not part of the experimental stimulus set. With each example, the experimenter described how it was possible to come up with a less negative interpretation of the picture content (e.g., a bloody crime scene could be seen as the place where a murder investigation was finally solved). The participants were then encouraged to generate reinterpretations for several other sample pictures. The participants reported their reinterpretations to the experimenter; these reinterpretations typically involved a story that resolved a seemingly negative picture in a less negative way (e.g., the man depicted in Figure 1 decided not to commit suicide). Once the experimenter determined that the participants were successfully reappraising negative pictures, the participants performed a practice block of 10 trials and then a block of 40 experimental trials. The 40 experimental trials consisted of the 40 unpleasant stimuli from the passive viewing block.

Figure 1 illustrates the experimental design and time course of the stimulus events in the emotion regulation block. Each trial began with the presentation of the word VIEW for $1,000 \mathrm{msec}$, followed by the 1,000-msec presentation of an unpleasant picture. These events were followed by a $4,500-\mathrm{msec}$ instruction to REAPPRAISE $(50 \%$ of the trials; the experimental condition) or a 4,500-msec instruction to ATTEND (50\% of the trials; the control condition). After this instruction, a blank screen followed for $500 \mathrm{msec}$, after which the unpleasant picture was again presented for $2,000 \mathrm{msec}$. The participants were instructed to simply view the first presentation of each unpleasant picture and to generate a less negative interpretation of the picture only after seeing the reappraise instruction. In contrast, the participants were instructed not to alter their natural feelings about pictures following the attend instruction. After the second presenta- 


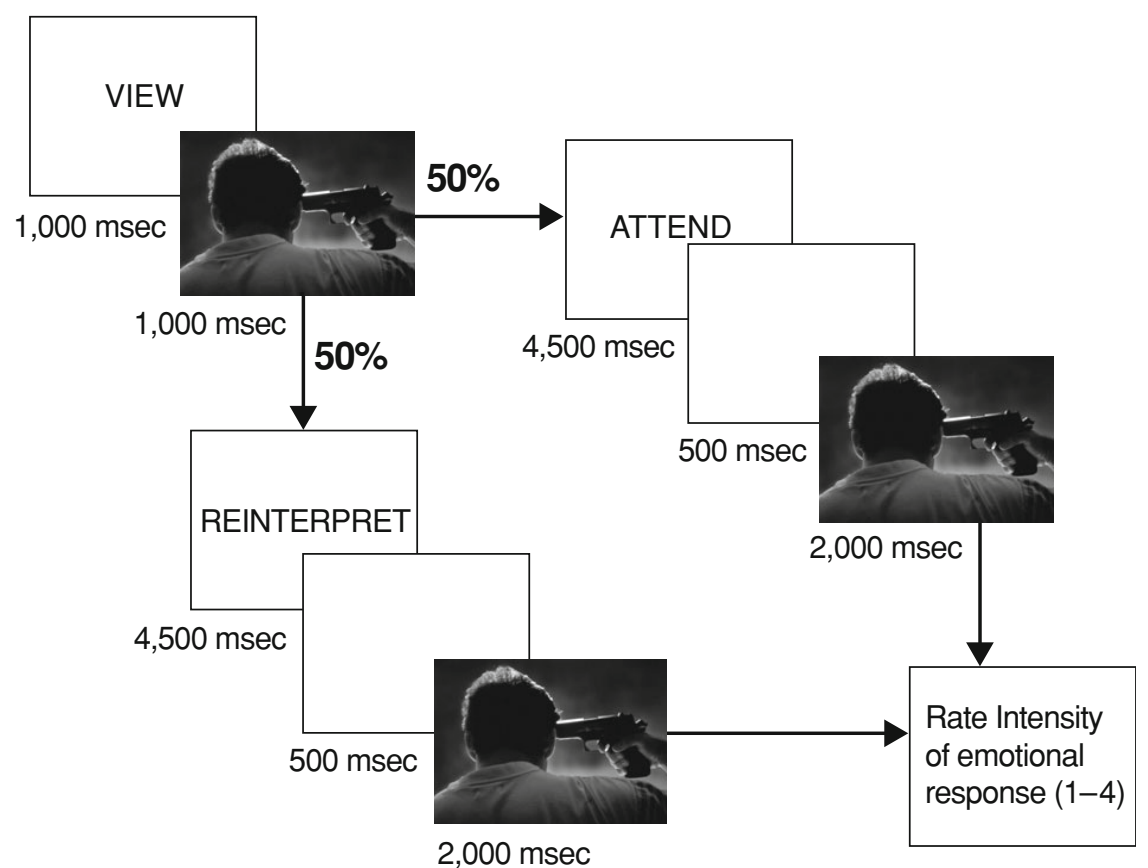

Figure 1. Experimental design and time course of stimulus events in the emotion regulation block. In the experiment, instructions were presented in white font on a black background.

tion of each unpleasant picture, the participants were asked to rate the intensity of their emotional response on a scale from 1 (weak) to 4 (strong). They were instructed to rate the intensity of their emotional response to only the second presentation of each picture. Finally, the order of the reappraise and attend trials was randomized, and the assignment of unpleasant pictures to the two conditions was counterbalanced across participants.

\section{Psychophysiological Recording, Data Reduction, and Analysis}

The electroencephalogram (EEG) was recorded with $32 \mathrm{Ag} / \mathrm{AgCl}$ electrodes embedded in a fabric cap (Electro-Cap International, Eaton, $\mathrm{OH}$ ) that was placed in an extended 10-20 montage and referenced to the left mastoid. Offline, all activity was re-referenced to the mean of the left and right mastoids. The electro-oculogram (EOG) was recorded with electrodes placed above and below the right eye and on the outer canthus of each eye. All impedances were kept below $10 \mathrm{k} \Omega$, and the EEG was amplified with SynAmps (band-pass filter $0.1-70 \mathrm{~Hz}$ ) and digitized at $250 \mathrm{~Hz}$.

Single-trial epochs were extracted offline for a period that started $100 \mathrm{msec}$ prior to picture onset and continued for the duration of stimulus presentation $(1,100 \mathrm{msec}$ total for the passive viewing block and 2,100 msec total for the emotion regulation block). Standard Neuroscan (Neurosoft Inc., Sterling, VA) analysis procedures were used to correct for EOG artifacts and to discard trials with recording artifacts. EEG epochs were then averaged to form stimulus-locked ERPs. Separate ERPs were computed for each picture type in the passive viewing block and for the unpleasant pictures that followed the reappraise and attend instructions. The resulting ERPs were baseline corrected and low-pass filtered $(<16 \mathrm{~Hz}, 12 \mathrm{~dB} /$ oct, zerophase shift). Scalp topography plots were created using EEGLAB (Delorme \& Makeig, 2004). For each participant, the amplitude of the LPP was defined as the average signal value at electrode $\mathrm{CPz}$ (where the LPP modulation was largest) in a time window that began $500 \mathrm{msec}$ after stimulus onset and continued for the duration of the recording epoch $(500-1,000 \mathrm{msec}$ in the passive viewing block; $500-2,000 \mathrm{msec}$ in the emotion regulation block).

\section{RESULTS}

\section{Passive Viewing Block}

Figure 2 (top) presents the ERP waveforms associated with pleasant, neutral, and unpleasant stimuli in the passive viewing block. Consistent with previous studies, emotionally salient stimuli elicited a large positive ERP modulation in comparison with neutral stimuli. This difference began approximately $250 \mathrm{msec}$ after stimulus onset and continued for the duration of the stimulus. As expected, the scalp topography of this LPP modulation had a focus over posterior midline sites (Figure 2, bottom). The magnitude of the LPP differed as a function of picture type $[F(2,24)=47.58, p<.001]$. Post hoc comparisons indicated that the LPPs associated with pleasant and unpleasant stimuli differed from the LPP associated with neutral stimuli $[t(13)=8.73, p<.001$, and $t(13)=$ $7.90, p<.001$, respectively], but not from each other $[t(13)=.66, p>.50]$. These results are consistent with those of previous studies that indicate that the LPP is sensitive to the emotional intensity but not to the valence of pictures. Furthermore, the $\mathrm{CPz}$ maximum of the LPP is consistent with the scalp topography of this component in several previous studies (Cuthbert et al., 2000; Keil et al., 2002; Schupp et al., 2000; Schupp et al., 2003).

\section{Emotion Regulation Block}

Emotional intensity ratings obtained in the emotion regulation block indicate that participants felt a less intense emotional response to unpleasant pictures that followed a reappraise instruction $(M=1.75, S D=.44)$ than to unpleasant pictures that followed an attend instruction 

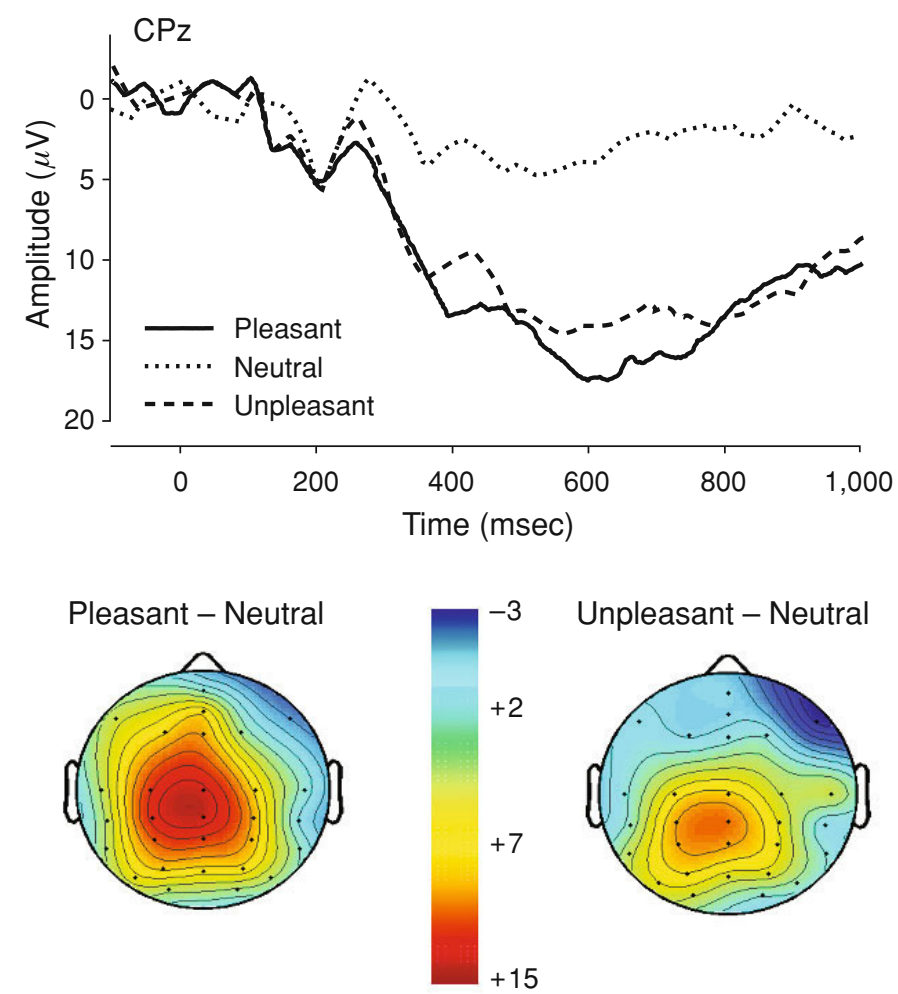

Figure 2. Top: ERP waveforms at electrode $\mathrm{CPz}$ associated with pleasant, neutral, and unpleasant stimuli in the passive viewing block. Note that negative voltage changes are plotted as upward deflections. Bottom: Voltage maps for the pleasant minus neutral and unpleasant minus neutral comparisons at $t=700 \mathrm{msec}$.

$(M=2.90, S D=.45 ; t(13)=6.80, p<.001]$. These results indicate that reappraisal successfully reduced the perceived emotional intensity of unpleasant stimuli.

Figure 3 (left) presents the ERP waveforms elicited by unpleasant stimuli in the reappraise and attend conditions. The figure shows that the magnitude of the LPP was substantially smaller in the reappraise condition than in the attend condition, and that this reappraisal-related LPP modulation started early ( 200 msec) after the onset of the unpleasant stimulus. The scalp topography of this modulation (see Figure 3, right) indicates a posterior midline focus similar to that of the basic LPP effect observed in the passive viewing block. ${ }^{2}$ The overall magnitude of the LPP was reliably reduced on reappraise trials $(M=3.56, S D=3.48)$ relative to attend trials $[M=$ $6.40, S D=3.16 ; t(13)=2.31, p<.05]$. To examine the time course of this difference in greater detail, we calculated the average voltage value for the attend and reappraise waveforms in consecutive 100 - $\mathrm{msec}$ windows, beginning with stimulus onset. The $100-\mathrm{msec}$ windows during which the reappraise and attend LPPs differed are indicated by the shaded gray areas in Figure 3. The reappraise LPP was reliably smaller than the attend LPP at 200-400 msec, 600-1,000 msec, and 1,200-1,800 msec after stimulus presentation (all $p \mathrm{~s}<.05$ ).
To examine whether the LPPs may have been subject to habituation or other changes associated with multiple stimulus presentations, we compared the LPPs elicited by unpleasant stimuli before the presentation of instructions with those elicited afterward. Although the LPP was somewhat reduced between the first $(M=9.20, S D=$ $6.37)$ and second $(M=6.40, S D=3.16)$ presentations on attend trials, this difference did not reach significance $[t(13)=1.82, p>.05]$. In contrast, on reappraise trials the LPP was reliably reduced from the first $(M=8.02$, $S D=4.21)$ to the second $(M=3.55, S D=3.48)$ presentation $[t(13)=3.91, p<.01]$. The LPPs elicited by unpleasant stimuli that preceded the reappraise and attend instructions did not differ from one another $[t(13)=.95$, $p>$.35]. Together, these results indicate that the systematic effects of habituation and other nonspecific factors on LPP magnitude were absent. Instead, they support the conclusion that the observed amplitude reduction of the reappraise LPP was largely due to the effects of the reappraise instruction.

Finally, the degree of LPP reduction following the reappraise instruction was positively related to reductions in self-reported emotional reactivity $[r(14)=.46, p<.05$; Figure 4], a fact which indicates that participants who evinced a large reduction in the LPP in the attend versus 


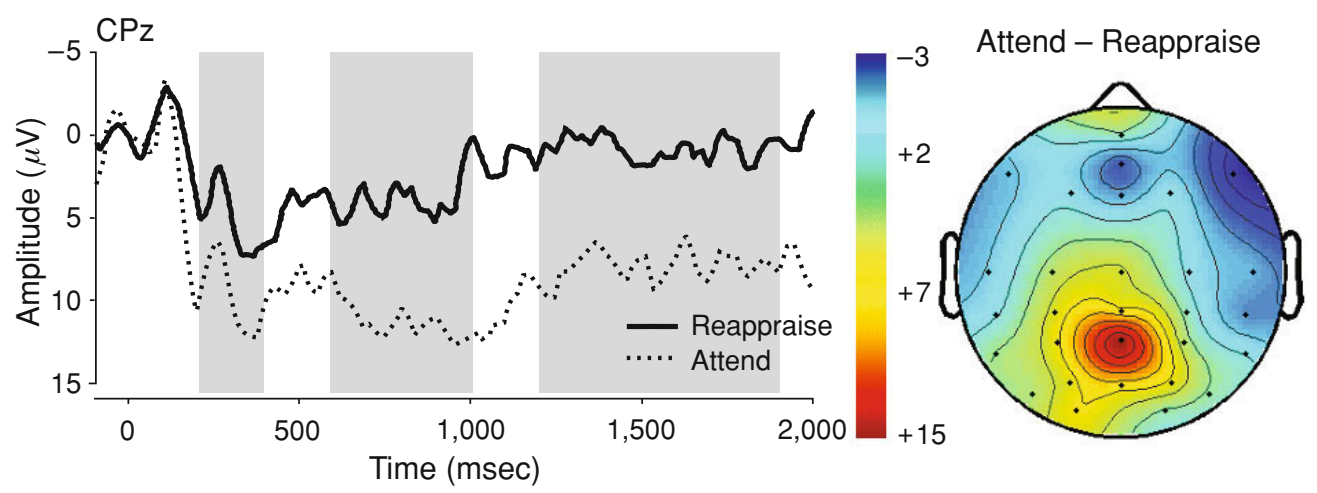

Figure 3. Left: ERP waveforms at electrode CPz associated with unpleasant stimuli in the reappraise and attend conditions of the emotion regulation block. Note that negative voltage changes are plotted as upward deflections. Shaded gray areas represent $100-\mathrm{msec}$ windows in which the reappraise LPP differed reliably from the attend LPP. Right: Voltage map for the attend minus reappraise comparison at $t=700 \mathrm{msec}$.

the reappraise instruction also tended to report a large reduction in emotional intensity following the reappraise instruction.

\section{DISCUSSION}

The present study provides further evidence that reappraisal can alter emotional responses to unpleasant stimuli. Going beyond previous studies, we established a distinct electrophysiological correlate of reappraisal-related modulation of emotional processing: Reappraisal was associated with a protracted reduction in the magnitude of the LPP, a slow-wave positive brain potential. More precise temporal analyses of this effect indicated that the reappraisal-induced LPP modulation began just $200 \mathrm{msec}$ after the onset of unpleasant stimuli. This suggests that reappraisal alters the very early neural response to unpleasant stimuli. In addition to beginning early, the reduction in the magnitude of the LPP continued for at least $2 \mathrm{sec}$, indicating that reappraisal influenced stimulus processing for the entire duration of stimulus presentation. These findings complement previous neuroimaging studies that have provided valuable information regarding the neural structures that are involved in reappraisal (Ochsner et al., 2002; Phan et al., 2005).

Consistent with previous studies, during passive viewing the LPP was enhanced after the presentation of both pleasant and unpleasant pictures as opposed to neutral pictures. In contrast, the magnitude of the LPP did not differ between pleasant and unpleasant pictures. These data are consistent with findings of previous studies indicating that the LPP is sensitive to the emotional intensity or motivational significance rather than the valence of emotional pictures (Cuthbert et al., 2000; Keil et al., 2002; Lang et al., 1997; Schupp et al., 2000; Schupp et al., 2003). When participants were asked to reappraise unpleasant pictures, a substantial reduction occurred in the amplitude of the LPP relative to that for the control condition, in which participants were asked to simply attend to the pictures. Furthermore, this re- duction in LPP amplitude was positively correlated with a reduction in the self-reported emotional intensity that followed reappraisal, a fact which indicates that there is a meaningful correlation between neural activity and phenomenological experience.

In terms of the underlying neural architecture that supports reappraisal, functional imaging studies have found that reappraisal of negative events is associated with increased activity in prefrontal brain areas involved in cognitive control and decreased activity in the amygdala and other brain areas involved in emotional response (Ochsner et al., 2002; Ochsner \& Gross, 2004; Phan et al., 2005). Similar results have been obtained in experiments that required participants to actively suppress their emotional responses (Beauregard et al., 2001; Lévesque et al., 2003) and in experiments in which participants made nonemotional as opposed to emotional decisions about affective stimuli (Hariri, Mattay, Tessitore, Fera, \& Weinberger, 2003; Keightley et al., 2003). Together, these results suggest that a similar network of brain regions is implicated in several emotion regulation techniques.

Mirroring the above-described functional imaging results, initial electrophysiological results suggest that the suppression of negative emotional responses to unpleasant pictures also reduces the magnitude of the LPP (Moser et al., 2006). In addition, in a recent study we have found that making nonemotional decisions about affective pictures reduces the magnitude of the LPP (Hajcak, Moser, $\&$ Simons, 2006). Thus, the available electrophysiological data indicate that a variety of emotion regulation techniques are reflected in reductions of the LPP.

It is important to note several limitations of the present study. First, the correlation between reductions in emotional intensity and LPP amplitude was based on average individual data; future research could further investigate the relationship between the LPP and emotion regulation by collecting emotional intensity ratings both before and after reappraisal instructions. This would allow for a within-subjects comparison to be made of LPP modulations related to large versus small changes in self-reported emotional intensity. 


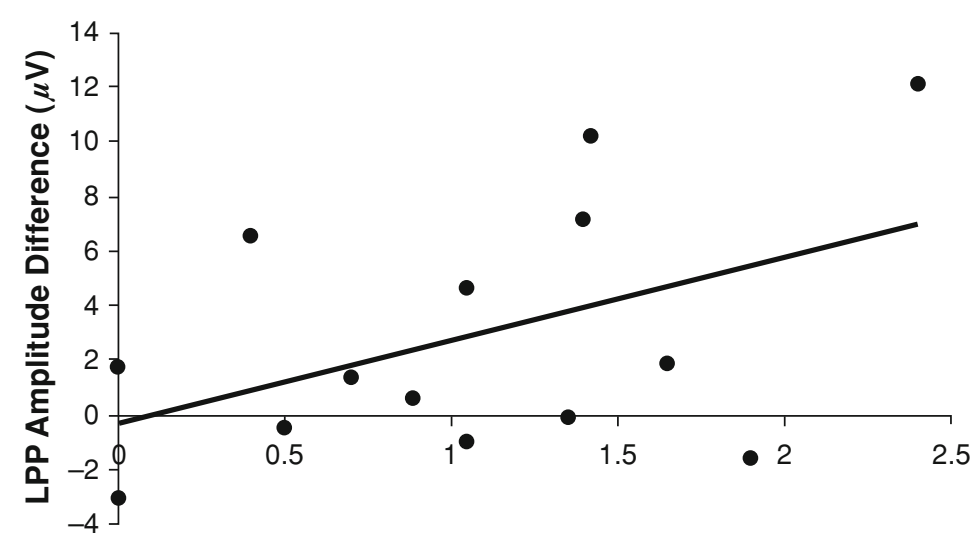

Self-Reported Emotional Intensity Difference

Figure 4. Change in LPP amplitude (attend minus reappraise) plotted as a function of change in self-reported emotional intensity (attend minus reappraise). Each dot represents an individual participant.

Second, the present study focused solely on the reappraisal of unpleasant images. We chose to focus on this specific process because it appears to be adaptive: Reappraisal has been shown to successfully reduce negative emotional responses to unpleasant stimuli (Gross, 2002); the tendency to use reappraisal has been related to measures of well-being (Gross \& John, 2003; John \& Gross, 2004); and this type of reappraisal is similar to the key aspects of cognitive therapy. Nonetheless, it remains an open question whether reappraisal would result in similar modulations of the LPP if the stimuli were pleasant. Measuring neurobiological changes associated with the up- and down-regulation of both positive and negative emotional responses (Ochsner et al., 2004) - preferably by combining functional imaging and electrophysiological measurements - will be important objectives for future research on emotion regulation.

Finally, it should be noted that our methods did not allow us to verify whether the reappraisal-related modulation of the LPP was a direct result of reappraisal per se. In principle, it is possible that on reappraise trials participants were doing something else to attenuate their emotional response (e.g., distracting themselves from the images). Asking participants to report their particular reappraisal on a trial-by-trial basis might help rule out this type of alternative explanation in future studies.

The present study raises a number of other important questions that can be addressed in future work. For example, is there a difference between the impact and the effectiveness of various types of reappraisal (cf. Ochsner et al., 2004)? An interesting avenue for future research would be to compare the LPP reductions associated with multiple emotion regulation strategies. Along similar lines, it would be informative to know whether people who use reappraisal strategies more frequently are better able to modulate their electrophysiological responses to emotional stimuli. Ultimately, this type of research would be most fruitful if it informed our understanding of psychopathology and its treatment. This research might involve utilizing the LPP to study emotional processing and emotion regulation in relationship to various personality traits (Ray et al., 2005) and in groups of patients with an impaired ability to regulate their emotions. Similarly, researching whether these neurobiological measures of emotion regulation change after cognitive therapy and other intervention methods are utilized will shed light on their etiological role in psychopathology.

\section{REFERENCES}

American Psychiatric Association (1994). Diagnostic and statistical manual of mental disorders: DSM-IV. Washington, DC: Author.

Anderson, A. K., \& Phelps, E. A. (2001). Lesions of the human amygdala impair enhanced perception of emotionally salient events. $\mathrm{Na}$ ture, 411, 305-309.

BeAuregard, M., LÉvesque, J., \& Bourgouin, P. (2001). Neural correlates of conscious self-regulation of emotion. Journal of Neuroscience, 21, 6993-7000.

CUThBert, B. N., Schupp, H. T., Bradley, M. M., Birbaumer, N., \& LANG, P. J. (2000). Brain potentials in affective picture processing: Covariation with autonomic arousal and affective report. Biological Psychology, 52, 95-111.

DELORME, A., \& MAKEIG, S. (2004). EEGLAB: An open source toolbox for analysis of single-trial EEG dynamics including independent component analysis. Journal of Neuroscience Methods, 134, 9-21.

Gross, J. J. (1998). The emerging field of emotion regulation: An integrative review. Review of General Psychology, 2, 271-299.

Gross, J. J. (2002). Emotion regulation: Affective, cognitive, and social consequences. Psychophysiology, 39, 281-291.

Gross, J. J., \& JoHN, O. P. (2003). Individual differences in two emotion regulation processes: Implications for affect, relationships, and wellbeing. Journal of Personality \& Social Psychology, 85, 348-362.

HAJCAK, G., MoSER, J. S., \& SimONS, R. F. (2006). Attending to affect: Appraisal strategies modulate the electrocortical response to arousing pictures. Emotion, 6, 517-522.

Hariri, A. R., Mattay, V. S., Tessitore, A., Fera, F., \& Weinberger, D. R. (2003). Neocortical modulation of the amygdala response to fearful stimuli. Biological Psychiatry, 53, 494-501.

JoHN, O. P., \& GROss, J. J. (2004). Healthy and unhealthy emotion regulation: Personality processes, individual differences, and life span development. Journal of Personality, 72, 1301-1333.

Keightley, M. L., WinOcur, G., GrahaM, S. J., Mayberg, H. S., 
Hevenor, S. J., \& Grady, C. L. (2003). An fMRI study investigating cognitive modulation of brain regions associated with emotional processing of visual stimuli. Neuropsychologia, 41, 585-596.

Keil, A., Bradley, M. M., Hauk, O., Rockstroh, B., Elbert, T., \& LANG, P. J. (2002). Large-scale neural correlates of affective pictureprocessing. Psychophysiology, 39, 641-649.

LANG, P. J., BRADLEY, M. M., \& CUTHBERT, B. N. (1997). Motivated attention: Affect, activation, and action. In P. J. Lang, R. F. Simons, \& M. T. Balaban (Eds.), Attention and orienting: Sensory and motivational processes (pp. 97-135). Mahwah, NJ: Erlbaum.

Lang, P. J., Bradley, M. M., \& Cuthbert, B. N. (1999). International Affective Picture System: Instruction manual and affective ratings (Tech. Rep. No. A-4). University of Florida, Center for Research in Psychophysiology.

LAZARUS, R. S. (1991). Progress on a cognitive-motivational-relational theory of emotion. American Psychologist, 46, 819-834.

Lévesque, J., Eugene, F., Joanette, Y., Paquette, V., Mensour, B., BeAudoin, G., ET AL. (2003). Neural circuitry underlying voluntary suppression of sadness. Biological Psychiatry, 53, 502-510.

Moser, J. S., Hajcak, G., Bukay, E., \& Simons, R. F. (2006). Intentional modulation of emotional responding to unpleasant pictures: An ERP study. Psychophysiology, 43, 292-296.

Ochsner, K. N., Bunge, S. A., Gross, J. J., \& Gabrieli, J. D. E. (2002). Rethinking feelings: An fMRI study of the cognitive regulation of emotion. Journal of Cognitive Neuroscience, 14, 1215-1229.

OChsner, K. N., \& Gross, J. J. (2004). Thinking makes it so: A social cognitive neuroscience approach to emotion regulation. In R. F. Baumeister \& K. D. Vohs (Eds.), Handbook of self-regulation: Research, theory, and applications (pp. 229-255). New York: Guilford.

Ochsner, K. N., Ray, R. D., Cooper, J. C., Robertson, E. R., Chopra, S., Gabrieli, J. D. E., \& Gross, J. J. (2004). For better or for worse: Neural systems supporting the cognitive down- and upregulation of negative emotion. NeuroImage, 23, 483-499.

Phan, K. L., Fitzgerald, D. A., Nathan, P. J., Moore, G. J., Uhde, T. W., \& TANCER, M. E. (2005). Neural substrates for voluntary suppression of negative affect: A functional magnetic resonance imaging study. Biological Psychiatry, 57, 210-219.

Ray, R. D., OChSner, K. N., CoOper, J. C., Robertson, E. R., GABRIELI, J. D. E., \& GRoss, J. J. (2005). Individual differences in trait rumination and the neural systems supporting cognitive reappraisal. Cognitive, Affective, \& Behavioral Neuroscience, 5, 156-168.

Schupp, H. T., Cuthbert, B. N., Bradley, M. M., CaCiopPo, J. T., ITO, T., \& LANG, P. J. (2000). Affective picture processing: The late positive potential is modulated by motivational relevance. Psychophysiology, 37, 257-261.

Schupp, H. T., Junghöfer, M., Weike, A. I., \& Hamm, A. O. (2003). Emotional facilitation of sensory processing in the visual cortex. Psychological Science, 14, 7-13.

THOMPSON, R. A. (1994). Emotion regulation: A theme in search of definition. Monographs of the Society for Research in Child Development, 59, 25-52, 250-283.

Whalen, P. J., Rauch, S. L., Etcoff, N. L., McInerney, S. C., Lee, M. B., \& JENIKE, M. A. (1998). Masked presentations of emotional facial expressions modulate amygdala activity without explicit knowledge. Journal of Neuroscience, 18, 411-418.

\section{NOTES}

1. The IAPS pictures used were pleasant $(1601,2000,2070,2080$, 2091, 2092, 2165, 2311, 2340, 4002, 4180, 4220, 4290, 4532, 4572, $4608,4658,4659,4660,4664,4800,4810,5470,5621,5626,5628$, $7325,8021,8032,8080,8200,8210,8280,8320,8330,8370,8400$, $8465,8490,8540)$; neutral $(2190,2480,2570,2840,2880,5390,5500$, $5510,5532,5534,5731,5740,5800,5900,7000,7002,7004,7006$, 7009, 7010, 7025, 7030, 7034, 7035, 7040, 7060, 7080, 7090, 7100, $7140,7150,7175,7190,7217,7224,7233,7235,7491,7950)$; and unpleasant $(1300,1301,2053,2120,2710,2800,2900,3160,3220,3230$, $3300,3350,3500,3530,6200,6210,6212,6230,6244,6250,6260$, $6312,6313,6370,6540,6550,6560,6570,6571,6821,9040,9050$, 9421, 9490, 9520, 9600, 9620, 9911, 9920, 9921).

2. Although there appears to be a midline frontal modulation in the topographical map in Figure 3, this modulation was not consistently present across participants.

(Manuscript received September 7, 2005; revision accepted for publication March 15, 2006.) 